\title{
Cyclic loss of open solar flux since 1868: the link to heliospheric current sheet tilt and implications for the Maunder Minimum
}

Article

Published Version

Owens, M. and Lockwood, M. (2012) Cyclic loss of open solar flux since 1868: the link to heliospheric current sheet tilt and implications for the Maunder Minimum. Journal of Geophysical Research - Space Physics, 117 (A4). ISSN 0148-0227 doi: https://doi.org/10.1029/2011JA017193 Available at https://centaur.reading.ac.uk/27767/

It is advisable to refer to the publisher's version if you intend to cite from the work. See Guidance on citing.

To link to this article DOI: http://dx.doi.org/10.1029/2011JA017193

Publisher: American Geophysical Union

All outputs in CentAUR are protected by Intellectual Property Rights law, including copyright law. Copyright and IPR is retained by the creators or other copyright holders. Terms and conditions for use of this material are defined in the End User Agreement.

www.reading.ac.uk/centaur 
Central Archive at the University of Reading

Reading's research outputs online 


\title{
Cyclic loss of open solar flux since 1868: The link to heliospheric current sheet tilt and implications for the Maunder Minimum
}

\author{
M. J. Owens ${ }^{1,2}$ and M. Lockwood ${ }^{1}$ \\ Received 23 September 2011; revised 16 January 2012; accepted 16 February 2012; published 3 April 2012.
}

[1] Open solar flux (OSF) variations can be described by the imbalance between source and loss terms. We use spacecraft and geomagnetic observations of OSF from 1868 to present and assume the OSF source, $S$, varies with the observed sunspot number, $R$. Computing the required fractional OSF loss, $\chi$, reveals a clear solar cycle variation, in approximate phase with $R$. While peak $R$ varies significantly from cycle to cycle, $\chi$ is surprisingly constant in both amplitude and waveform. Comparisons of $\chi$ with measures of heliospheric current sheet (HCS) orientation reveal a strong correlation. The cyclic nature of $\chi$ is exploited to reconstruct OSF back to the start of sunspot records in 1610. This agrees well with the available spacecraft, geomagnetic, and cosmogenic isotope observations. Assuming $S$ is proportional to $R$ yields near-zero OSF throughout the Maunder Minimum. However, $\chi$ becomes negative during periods of low $R$, particularly the most recent solar minimum, meaning OSF production is underestimated. This is related to continued coronal mass ejection (CME) activity, and therefore OSF production, throughout solar minimum, despite $R$ falling to zero. Correcting $S$ for this produces a better match to the recent solar minimum OSF observations. It also results in a cycling, nonzero OSF during the Maunder Minimum, in agreement with cosmogenic isotope observations. These results suggest that during the Maunder Minimum, HCS tilt cycled as over recent solar cycles, and the CME rate was roughly constant at the levels measured during the most recent two solar minima.

Citation: Owens, M. J., and M. Lockwood (2012), Cyclic loss of open solar flux since 1868: The link to heliospheric current sheet tilt and implications for the Maunder Minimum, J. Geophys. Res., 117, A04102, doi:10.1029/2011JA017193.

\section{Introduction}

[2] Open solar flux (OSF) is the component of the coronal magnetic field which is dragged out into the heliosphere by the solar wind. Cosmogenic isotope records in ice cores, ocean sediments and tree trunks provide a means of inferring the prehistoric OSF, which in turn can be used to reconstruct the photospheric field and, ultimately, solar irradiance variations needed for long-term climate modeling [e.g., Wang et al., 2005; Lockwood, 2006; Krivova et al., 2007; Steinhilber et al., 2009, and references therein]. In addition, the OSF variation is key to understanding past and future space weather conditions at Earth [Barnard et al., 2011]. Determining the mechanisms by which OSF evolves over the solar cycle is vital for understanding, reconstructing and predicting long-term variations in solar and heliospheric magnetism. Solanki et al. [2000] describe OSF as a continuity equation:

$$
\frac{d(\mathrm{OSF})}{d t}=S-L
$$

\footnotetext{
${ }^{1}$ Space Environment Physics Group, Department of Meteorology, University of Reading, Reading, UK.

${ }^{2}$ Space and Atmospheric Physics, Blackett Laboratory, Imperial College London, London, UK.

Copyright 2012 by the American Geophysical Union. 0148-0227/12/2011JA017193
}

where $S$ is the OSF source term and $L$ is the loss term. See also Table 1. The source of new OSF has to be magnetic loops being dragged out into the heliosphere from below the solar wind formation height. Clearly, this must be ultimately, albeit indirectly, related to magnetic flux emerging through the photosphere. Consequently, models of OSF evolution have successfully related $S$ to sunspot number, $R$ [Solanki et al., 2000; Krivova et al., 2007; Vieira and Solanki, 2010]. Owens and Crooker [2006] and Owens et al. [2008b, 2011a] argued that this approach is successful because $R$ is a proxy for the CME rate [Webb and Howard, 1994], which provide the actual mechanism by which OSF increases. Regardless, it is clear that $S$ has a strong solar cycle variation.

[3] The supersonic nature of the solar wind means that the loss of OSF must involve magnetic restructuring below the Alfven point, with two open solar flux tubes reconnecting to "disconnect" flux from the Sun (though this need not produce completely disconnected flux in the heliosphere [Crooker et al., 2002; Owens and Crooker, 2007]). The process(es) which control $L$ are not well understood, making it difficult to relate this parameter to observations. In the absence of any better physical constraint, some models have assumed that $L$ is constant [Connick et al., 2011]. However, it is more commonly assumed that OSF decays with time, such that $L(t)=\chi \operatorname{OSF}(t)$. The fractional OSF loss rate, $\chi$, is generally assumed to be constant [Solanki et al., 2000; Vieira and Solanki, 2010]. However, it has recently been suggested that $\chi$ is related to the 
Table 1. List of Symbols and Abbreviations Used in This Study

\begin{tabular}{ll}
\hline Symbol & Meaning \\
\hline OSF & Total unsigned open solar flux \\
$S$ & OSF source rate \\
$L$ & OSF loss rate \\
$\chi$ & Fractional OSF loss rate. i.e., \\
& $L=\chi$ OSF \\
$R_{Z}$ & Zurich/International monthly \\
& sunspot number \\
$R_{G}$ & Group monthly sunspot number \\
$R$ & Composite sunspot number used \\
& in this study \\
$\phi$ & Magnetic flux per sunspot per year. \\
& $\phi=13 \times 10^{12}$ Wb y ${ }^{-1}$ \\
$S_{0}$ & OSF source rate assuming $S_{0}=\phi R$ \\
$S_{10}$ & OSF source rate assuming \\
& $S_{10}=\phi(R+10)$ \\
$\chi_{0}$ & Fractional OSF loss rate required \\
& to match $S_{0}$ \\
$\chi_{10}$ & Fractional OSF loss rate required \\
$i_{H C S}$ & to match $S_{10}$ \\
$\Phi$ & Heliospheric current sheet tilt index \\
\hline
\end{tabular}

inclination of the heliospheric current sheet (HCS), which varies substantially over the solar cycle [Owens et al., 2011a].

[4] In section 2 we use OSF reconstructions back to 1868 , and assume that OSF production rate can be quantified using $R$, in order to compute the required OSF loss term. In section 4 , we compare the fractional OSF loss with the solar cycle variation in the HCS orientation. Finally, in section 5, we use the cyclic variation of $\chi$ with sunspot number records to reconstruct OSF back to 1610 .

\section{OSF Loss Rate Required to Match Observations}

[5] The open solar flux data used in this study are a composite of three time series. Space-age data (1965-2011) are based upon in situ near-Earth measurements of the radial magnetic field, $B_{R}[$ Owens et al., 2008a; Lockwood and Owens, 2009], kinematically corrected to allow for the effect of longitudinal structure in the solar wind flow during propagation from the solar source surface to the Earth [Lockwood et al., 2009a]. Prior to the space age, OSF estimates are based on geomagnetic index data using the method of Lockwood et al. [2009b]. Note that the number of independent geomagnetic measurements used to derive OSF drops significantly the further back in time the reconstruction is performed: From approximately 500 during 1930-2011, down to around 100-200 from 1900 to 1920. Before 1900, only a single geomagnetic station is available, providing a maximum of 24 independent hourly geomagnetic measurements. Thus prior to 1904 , the method of Lockwood et al. [1999] is used to compute the nearEarth IMF field strength, which is then converted to OSF using the polynomial fit presented by Lockwood and Owens [2011]. The periods of overlap of these three OSF data series show that agreement between them is very close [Lockwood and Owens, 2011].

[6] In order to remove the effect of ecliptic inclination (and in the case of OSF derived from geomagnetic data, the Earth's rotational axis tilt), data are computed at annual averages. Note also that for direct comparison with the work by Owens et al. [2011a], we define OSF as the total unsigned magnetic flux in the heliosphere, equal to $4 \pi r^{2}\left|B_{r}\right|$, where $r$ is the heliocentric distance and $B_{r}$ is the radial component of the heliospheric magnetic field. Thus the OSF values are precisely twice the total signed OSF used by Lockwood and Owens [2011]. This OSF time series is shown as the dark shaded region in Figure 1.

[7] Sunspot number is used as a proxy for the OSF production rate. Where possible, we use group sunspot number, $R_{G}$ [Hoyt and Schatten, 1998], as it represents a more complete record than Zurich/International sunspot, $R_{Z}$, particularly prior to 1850 [Hathaway et al., 2002]. We incorporate the recent $R_{G}$ correction to observations from 1637 to 1642 [Vaquero et al., 2011]. After 1995 , when $R_{G}$ is not available, we use $R_{Z}$. The blue lines in Figure 1 show this composite sunspot time series, hereafter denoted by $R$, scaled down by a factor 15 to fit on the same plot y axis as OSF (the latter in $10^{14} \mathrm{~Wb}$ ). Figure 1 (top) shows the yearly $R$ and OSF data, and Figure 1 (bottom) shows solar cycle averages, taking the start of a cycle to be the time of the sharp increase in average sunspot latitude, which is concurrent with the minimum in sunspot number at this annual resolution [Owens et al., 2011b].

[8] Using these two sets of independent observations, it is possible to compute the required fractional OSF loss rate, $\chi$, from equation (1):

$$
\chi(t)=\frac{1}{\operatorname{OSF}(t)}\left[S(t)-\frac{\operatorname{OSF}(t+1)-\operatorname{OSF}(t)}{\Delta t}\right]
$$

where $t$ refers to the current time step and $\Delta \mathrm{t}$ is the time resolution of the data, in this case 1 year.

[9] In the first instance, we take the OSF source term, $S$, to be directly proportional to $R$, so that $S_{0}=\phi R$. We fix $\phi$ at $1 \times 10^{12} \mathrm{~Wb} \mathrm{CR}^{-1}$ (where CR is Carrington rotation) $\simeq 13 \times 10^{12} \mathrm{~Wb} \mathrm{yr}^{-1}$, as per Owens et al. [2011a], though the results presented here are qualitatively unaffected by this value of $\phi$. The corresponding fractional OSF loss rate, $\chi_{0}$, is shown as red lines in Figure 1, scaled up by a factor 3 in order to display on the same y axis. Addressing first the yearly data (Figure 1, top), there are a number of points of interest. First, given that $\chi_{0}$ is a series of independent values subject to noise in both the OSF and $R$ observations, it is surprisingly well ordered. Second, there is a very clear solar cycle variation in $\chi_{0}$, which emerges wholly from the data, rather than being a feature included in the model. At this yearly time resolution, $\chi_{0}$ appears to vary approximately in phase with $R$. There are a few points, clustered around solar minimum, where $\chi_{0}$ becomes slightly negative, which are discussed further below. Finally, we note that $\chi_{0}$ exhibits very little cycle-to-cycle variation in magnitude compared with $R$ and OSF. This is better displayed in the solar cycle averages of Figure 1 (bottom). While the cycle averages of $R$ roughly track those of OSF, $\chi_{0}$ remains approximately constant throughout the period, particularly after 1910. It is likely that this slight change is associated with the measurement uncertainty in the OSF data series, as the number of geomagnetic measurements used to generate the OSF changes significantly around this time, from 309 in 1930, to 95 in 1910 [e.g., Lockwood et al., 2009b].

[10] Figure 2 shows composites of OSF, $R$, and $\chi_{0}$ over all solar cycles since 1868. Data are shown as a function of solar cycle phase, rather than time, to remove the effect of variable solar cycle length. In the first row, OSF shows a solar cycle variation, but there is a great deal of cycle-to-cycle variation in the magnitude, largely because of the strong persistence in the time series on 

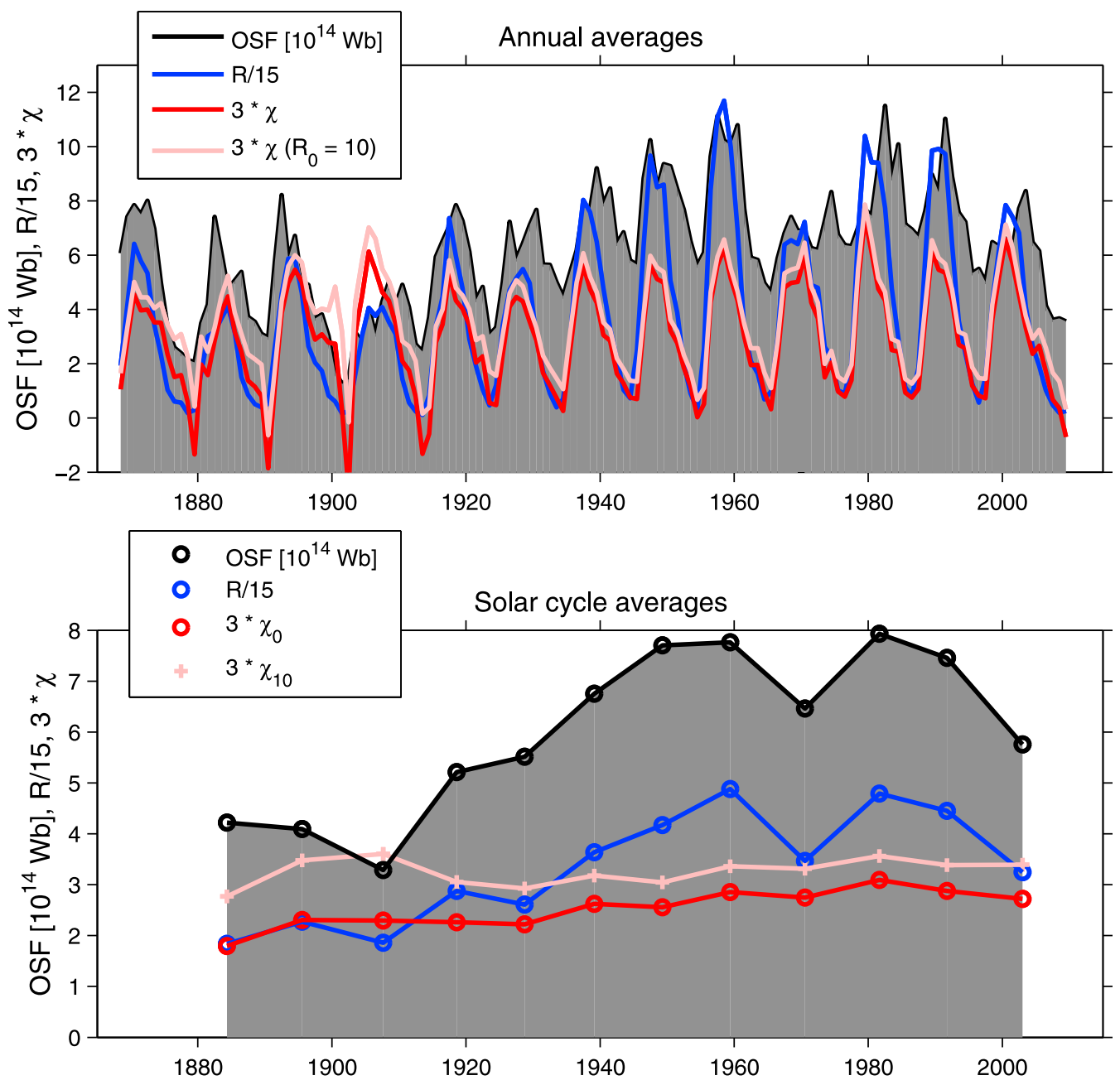

Figure 1. The evolution of open solar flux (OSF) since 1868 on (top) annual and (bottom) solar cycle time scales. The dark shaded areas show the total unsigned OSF inferred from spacecraft and geomagnetic observations, in units of $10^{14} \mathrm{~Wb}$. Blue lines shows the sunspot number, $R$, scaled down by a factor 15 . Red (pink) lines show $\chi_{0}\left(\chi_{10}\right)$, the required fractional OSF loss rate when the OSF production rate is assumed to be proportional to $R$ (proportional to $[R+10]$ ), scaled up by a factor 3 .

decadal time scales. In the second row, the waveform of $R$ is consistent from cycle to cycle, but the amplitude varies significantly. In the third row, $\chi_{0}$ shows significantly less cycle-to-cycle variation in magnitude than either OSF or $R$. The waveform also shows little variation. It is similar to, but slightly more asymmetric than, the $R$ waveform, with a sharper rise and a longer decline.

\section{Better Determination of the OSF Source Term}

[11] As can be seen in both Figures 1 and 2, a number of instances of negative $\chi_{0}$ occur at solar minimum, including the most recent minimum. This is equivalent to an additional OSF source term. Therefore these are periods when OSF production must be higher than suggested by the assumption of $S_{0} \propto R$. Figure 3 shows the relation between CME rate, a known source of new OSF [Gosling et al., 1987; Low, 2001], and sunspot number, $R$. CME rate data is taken from Large Angle and Spectrometric Coronagraph (LASCO) and STEREO coronagraph observations [St. Cyr et al., 2000; Yashiro et al., 2004; Gopalswamy et al., 2008], combined in the same manner as Owens et al. [2008b]. Figure 3 (top) shows time series of data averaged over one Carrington rotation (i.e., 27.27 days). Figure 3 (middle) shows a scatterplot of the same data, with the rainbow colors identifying the phase of the solar cycle. Figure 3 (bottom) shows data binned by $R$. Horizontal lines show the mean (solid) and error bars (dashed) in the average CME rate over the 14 Carrington rotations for which $R<2$. The CME rate varies approximately linearly with $R$ during much of the cycle [Webb and Howard, 1994]. Note that at solar minimum CME rate does not drop below 0.55 day $^{-1}$ [Owens et al., $2008 \mathrm{~b}$, while $R$ tends to zero. Also note that there may be a small difference in the rise and declining phase CME rates for the same $R$. Without more solar cycles of data, it is not clear whether this is a regular solar cycle feature or the result of a longer-term change in solar behavior superposed on top of the solar cycle variation. For this study, it is necessary to assume that the relation between $\mathrm{CME}$ rate and $R$ is approximately invariant over the solar cycle.

[12] A "square-root law" relation has been found between the $10.7 \mathrm{~cm}$ solar radio flux (F10.7), known to vary closely with $R$, and the intensity of Lyman $\alpha$ radiation, a proxy chromospheric magnetic fields [Woods et al., 2000]. Thus it might 
(a)

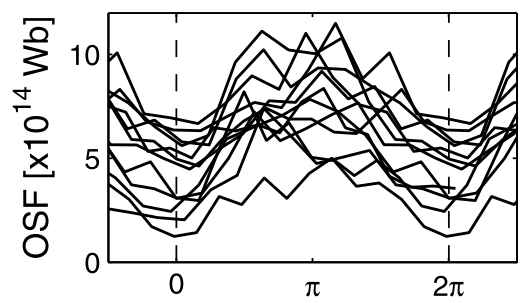

Solar cycle phase

(b)

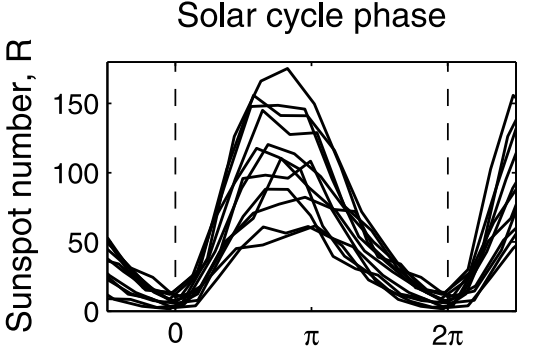

Solar cycle phase

(c)

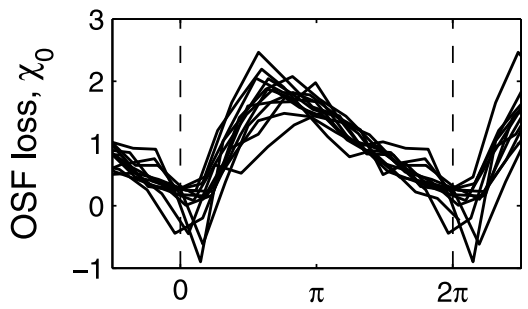

Solar cycle phase

(d)

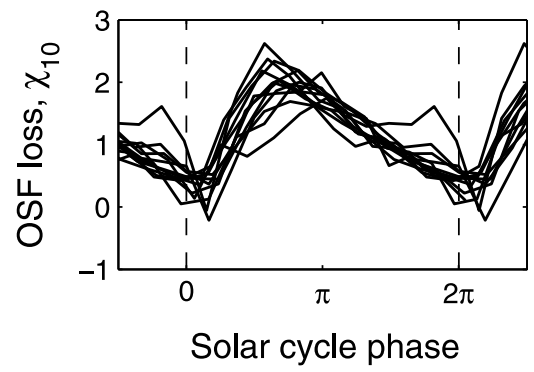

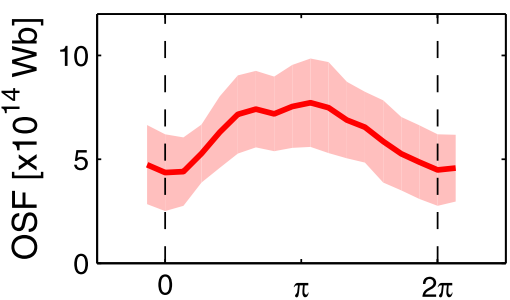

Solar cycle phase

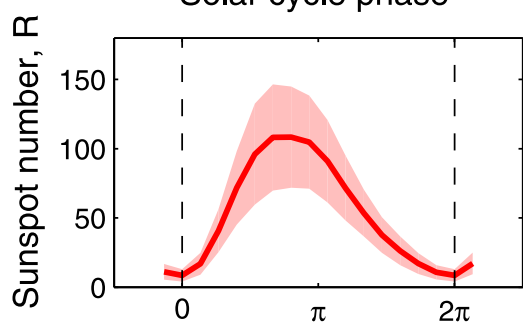

Solar cycle phase

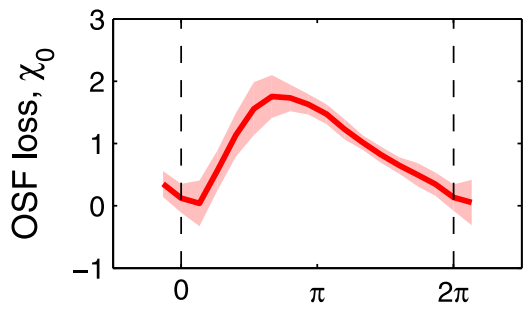

Solar cycle phase

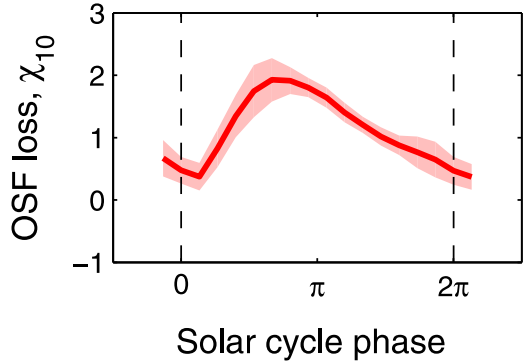

Figure 2. The solar cycle variations in (a) OSF, (b) sunspot number, $R$, and (c and d) fractional OSF loss, $\chi$. Figure $2 \mathrm{c}$ (Figure $2 \mathrm{~d}$ ) shows $\chi_{0}\left(\chi_{10}\right)$, the required fractional OSF loss rate when the OSF production rate is assumed to be proportional to $R$ (proportional to $[R+10]$ ). (left) The 13 cycles considered in this study; (right) the mean (thick red line) and \pm 1 standard deviation (pink shaded area). As seen in Figure 1, OSF and $R$ show considerably more cycle-to-cycle variation than OSF loss. Also, the waveform of $\chi$ is more asymmetric than those of $R$ and OSF.

be expected that CME rate and $R$ should obey a similar scaling. However, this would require zero CME rate at $R=0$, contrary to the observations from the recent solar minimum, particularly during much of 2009. Instead, the solid (dashed) black curves show the best fit (one sigma error bars) of a second-order polynomial. These imply the CME rate is 0.55 day $^{-1}$ for $R=0$, as observed. Extrapolating the fit back beyond that $R=0$ gives a zero CME rate at $R \simeq-10$. Thus CME rate is approximately proportional to $(R+10)$.

[13] In order to incorporate continued OSF production at $R=0$, we modify the OSF source term to $S_{10}=\phi(R+10)$. Note that we do not use the full quadratic fit between CME rate and $R$, as there are large uncertainties in the average CME rates at high $R$ because data are all from a single solar cycle. Additionally, there is much greater scatter in the CME rate- $R$ relation at high $R$. The fractional OSF loss rate required to match $S_{10}, \chi_{10}$, is shown as the pink line in Figure 1, and in Figures $2 \mathrm{c}$ and $2 \mathrm{~d}$. $\chi_{10}$ is largely unchanged from $\chi_{0}$, though it does remain above zero at almost all times.

\section{Relation to OSF Loss to HCS Inclination}

[14] Figure 4 compares the fractional OSF loss rate with estimates of heliospheric current sheet (HCS) tilt angle. The dark shaded region shows the potential field source surface (PFSS) [Schatten et al., 1969; Altschuler and Newkirk, 1969] estimate of the HCS tilt using Wilcox Solar Observatory (WSO) magnetograms. It is the inclination index $i_{H C S}$, a measure of the length of the HCS perpendicular to the rotation direction [Owens et al., 2011a], scaled up by a factor 1200, 

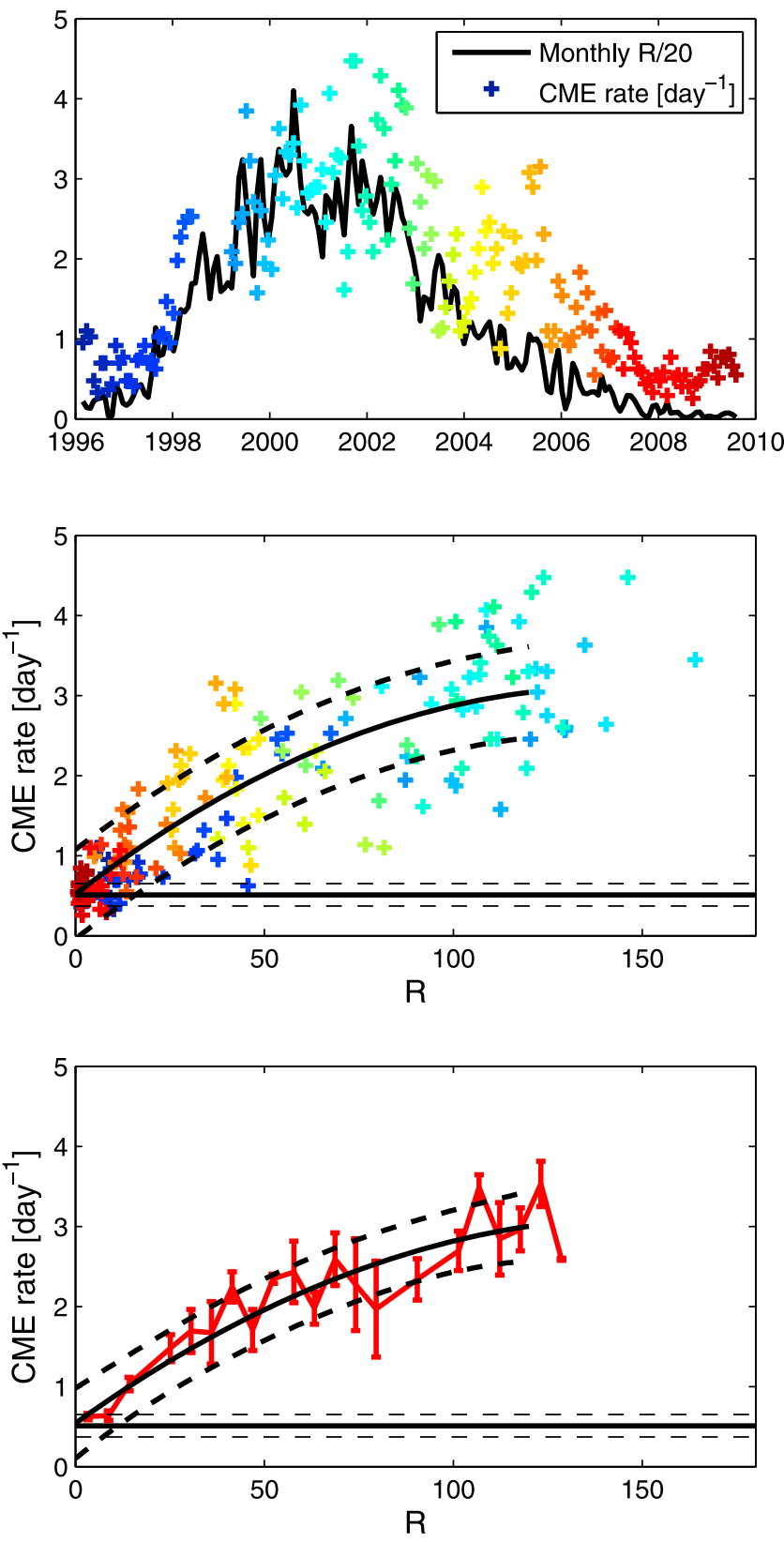

Figure 3. The relation between coronal mass ejection (CME) rate and sunspot number, $R$, over solar cycle 23. (top) Time series of Carrington rotation (CR) averages of $R$ and Large Angle and Spectrometric Coronagraph (LASCO) and STEREO derived CME rates. The rainbow colors are used to identify solar cycle phase in the subsequent scatterplots. (middle) A plot of CR averages of CME rate with $R$. (bottom) The same data, binned by $R$. Horizontal lines show the mean (solid) and error bars (dashed) in the average CME rate over the 14 Carrington rotations for which $R<2$. The solid (dashed) black curves show the best quadratic fit (one sigma error bars). Note that at solar minimum, CME rate does not drop below 0.55 day $^{-1}$, while $R$ tends to zero.

which agrees very closely with the "classic" PFSS estimate of HCS tilt angle (e.g., http://wso.stanford.edu/Tilts.html). The blue line shows the HCS tilt inferred from observed galactic cosmic ray (GCR) fluxes, described further below. The red and pink lines show the fractional OSF loss terms, $\chi_{0}$ and $\chi_{10}$, scaled up by a factor 30 .

[15] There is general good agreement between the solar cycle variations in $\chi$ and the PFSS estimate of HCS orientation. Much like $\chi$, HCS orientation shows little variation in the peak amplitude from cycle-to-cycle, despite the associated variations in cycle lengths and peak sunspot values [cf. Owens et al., 2011b]. Note that OSF loss in the declining phase of solar cycle 23 ( 2006-2010) better follows the HCS tilt when the $S_{10}$ correction is used. This is significant because this interval contains the recent "exceptional" solar minimum when solar activity returned to the lowest average levels seen since about 1900 and OSF fell approximately halfway back to Maunder Minimum levels [Lockwood, 2010]. Hence the better agreement of $S_{10}$ means it is more likely to reproduce Maunder Minimum conditions.

[16] The GCR-derived HCS tilt angle is obtained by modeling the heliospheric modulation parameter derived from neutron monitor observations [Alanko-Huotari et al., 2007]. Again, the agreement with $\chi$ is generally good. The most notable exception is solar cycle $20(\sim 1976-1987)$, but here the GCR-based estimates of HCS tilt also disagree with the PFSS estimates, which suggests GCR modulation may be more complex at this time [e.g., Ferreira and Potgieter, 2003]. Indeed, Alanko-Huotari et al. [2007] conclude that the heliospheric modulation parameter since 1951 is suggestive of HCS tilt varying only as a function of solar cycle phase, with little cycle-to-cycle variability (as also deduced here).

\section{Modeling OSF}

[17] We now use the cyclic variation in OSF loss, outlined in section 2 and linked with HCS orientation in section 4, to reconstruct the OSF back to the start of sunspot records in 1610. In essence, we use the model of Owens et al. [2011a], but the OSF loss term, originally based on the PFSS-derived HCS inclination, is replaced with the average behavior of $\chi$ obtained in section 2. Otherwise the model is unchanged, with no further parameterization.

[18] At each 1 year time step, solar cycle phase is used to determine the value of $\chi_{0}$ (or $\chi_{10}$ ). From 1870 to 2010, solar cycle start and end times are based on sunspot latitude [Owens et al., 2011b], before that, minima in $R$ are used. During the Maunder Minimum, when there are essentially no sunspots for extended periods, the maxima in ${ }^{10} \mathrm{Be}$ fluxes [Beer et al., 1998] are used, as done by Lockwood and Owens [2011]. At 1610, we take an arbitrary value for OSF of $5 \times 10^{14} \mathrm{~Wb}$, but the high turnover time for OSF means the choice of this value has no effect on OSF estimates past about 1615.

[19] The dark shading in Figure 5 (top) shows the reconstructed OSF back to 1610 , the start of the $R_{G}$ records. As might be expected, there is very close correspondence with the Vieira and Solanki [2010] OSF reconstruction (not shown, to avoid plot clutter), also based on sunspot number. Spacecraft and geomagnetic OSF observations, available back to 1868 , are shown in green. The red crosses (circles) in Figure 6 show the comparison between the observed and modeled OSF for the whole 1858-2011 interval (1910-2011, when the uncertainty in the observed OSF is significantly reduced): The agreement is very good, with a linear correlation coefficient of $0.879(0.857)$, significant at the $99.9 \%$ level. 


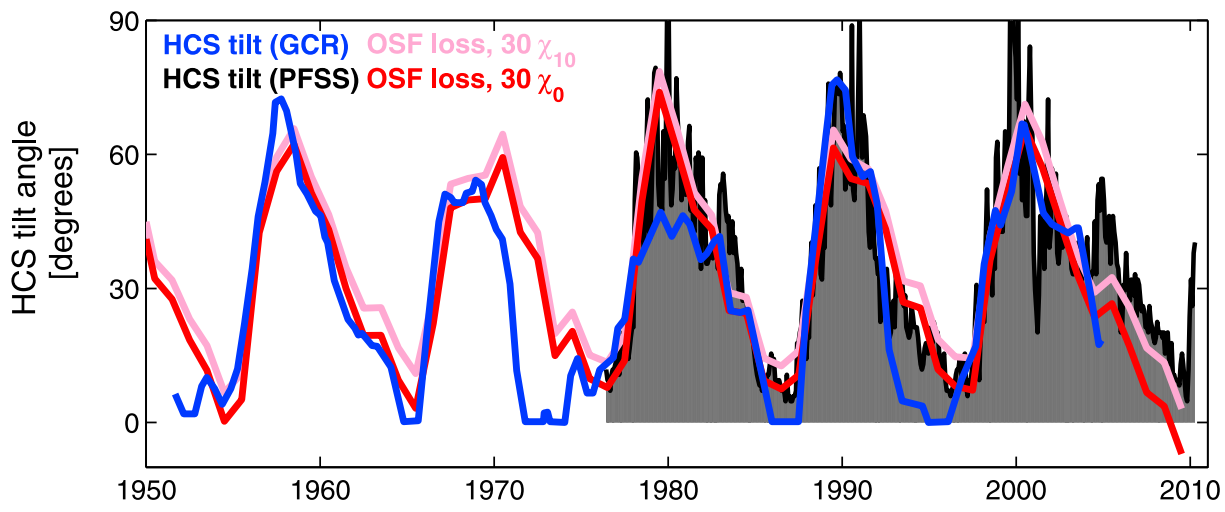

Figure 4. The fractional OSF loss rate compared with estimates of heliospheric current sheet orientation. The dark shaded region shows the heliospheric current sheet (HCS) tilt from potential field source surface (PFSS) modeling of Wilcox Solar Observatory (WSO) magnetograms. The blue line shows HCS tilt computed from modeling galactic cosmic ray observations. The red (pink) line shows $\chi_{0}\left(\chi_{10}\right)$ scaled up by a factor 30 . Fractional OSF loss is well correlated with HCS orientation.

[20] In order to compare our OSF model with observations prior to 1858 , cosmogenic isotope data must be used. Abundances of ${ }^{14} \mathrm{C}$ [Muscheler et al., 2007; Usoskin, 2008] and ${ }^{10} \mathrm{Be}$ [Usoskin et al., 2003; Usoskin, 2008; McCracken and Beer, 2007; McCracken, 2007] found in terrestrial reservoirs (tree trunks and ice sheets, respectively) have been used to infer the heliospheric modulation potential, $\Phi$. These $\Phi$ estimates $\left(\Phi_{14 C}\right.$ and $\left.\Phi_{10 B e}\right)$ have here been extended to recent years using $\Phi$ from neutron monitor data [e.g., Usoskin et al., 2005]. Comparison of $\Phi$ with geomagnetic values of OSF since 1868 reveals the following linear relations:

$$
\begin{aligned}
& \mathrm{OSF}_{14 \mathrm{C}}\left[\times 10^{14} \mathrm{~Wb}\right]=0.0086 \Phi_{14 C}+0.948 \\
& \mathrm{OSF}_{10 \mathrm{Be}}\left[\times 10^{14} \mathrm{~Wb}\right]=0.0098 \Phi_{10 B e}+1.92
\end{aligned}
$$

Both relations are significant at the $99 \%$ level, accounting for persistence in the time series using Monte Carlo iterations with frequency or time domain time series modeling [Macias Fauria et al., 2012]. However, such linear relations may slightly overestimate OSF as $\Phi$ tends toward zero, therefore we also use third-order polynomial fits, constrained to pass through $O S F=0$ at $\Phi=0$ [Lockwood and Fröhlich, 2008]:

$$
\begin{aligned}
\mathrm{OSF}_{14 \mathrm{C}}\left[\times 10^{14} \mathrm{~Wb}\right] & =-4.87 \times 10^{-6} \Phi_{14 C}^{2}+0.0090 \Phi_{14 C} \\
\mathrm{OSF}_{10 \mathrm{Be}}\left[\times 10^{14} \mathrm{~Wb}\right] & =-2.65 \times 10^{-6} \Phi_{10 B e}^{2}+0.0074 \Phi_{10 B e}
\end{aligned}
$$

These relations are again significant above the $99 \%$ level.

[21] Blue and red lines in Figure 5 show 10 year averages of OSF using ${ }^{14} \mathrm{C}$ and ${ }^{10} \mathrm{Be}$ data, respectively. OSF derived from linear fits is shown as solid lines, third-order polynomials as dashed lines. The long-term trends between these OSF estimates and our model are in reasonable agreement, though cosmogenic isotope data generally suggests nonzero OSF throughout the Maunder Minimum, while our model with OSF source term $S_{0}=\phi R$, like that of Vieira and Solanki [2010], predicts near-zero values.

[22] Figure 5 (bottom) shows an OSF reconstruction using $S_{10}=\phi(R+10)$. This change is introduced principally because over the last two solar minima, CME rates (and hence OSF emergence) are not observed to fall to zero when sunspot number, $R$ has fallen to zero. The new source term only significantly alters the reconstructed OSF at very limited times.
One such period is the most recent solar minimum, where the agreement between model and observation is improved. However, we also note that the error increases slightly during the 1901 minimum. Figure 6 shows that the $S_{0}$ and $S_{10}$ correlation coefficients between model and observed OSF are essentially equal over the whole 1868-2011 period, but higher for $S_{10}$ since 1910, when the observational uncertainty is reduced. Note also that the $S_{10}$ regression line has a slope closer to unity and a smaller zero offset than $S_{0}$, suggesting it provides the better match to the observations.

[23] Returning to Figure 5, the most obvious effect of using $S_{10}=\phi(R+10)$, rather than $S_{0}=\phi R$, is to increase OSF during the Dalton minimum (about 1800-1830) and, most prominently, the Maunder Minimum (about 1650-1715). Whereas the $S_{0}$ OSF dropped to zero during most of the Maunder Minimum, because $S \approx 0$ at this time, the $S_{10}$ modification introduces a near-constant source term during this period. Combined with the cyclic loss term, this produces a clear solar cycle variation in OSF. However, unlike the space age cycles, OSF peaks when the HCS is flat (i.e., solar minimum) and minimizes when the HCS is most inclined (i.e., solar maximum).

\section{Discussion and Conclusions}

[24] In this study, we have computed the open solar flux (OSF) loss required to match the observed OSF variation since 1868 , assuming the OSF production rate varies linearly with sunspot number, $R$ (as used, for example, by Solanki et al. [2000]). We find the fractional OSF loss rate, $\chi$, has a very strong solar cycle variation, approximately in phase with the $R$ variation, though with a more asymmetric waveform. Also of note is the relative constancy of both the amplitude and waveform of $\chi$ from cycle to cycle, compared with equivalent variations in $R$ and OSF. This is notably true for the variation of $\chi$ deduced after about 1910, when the OSF reconstruction is based on data from a greater number of geomagnetic observatories.

[25] It has been suggested that OSF loss is controlled by the orientation of the heliospheric current sheet (HCS), particular its inclination relative to the solar rotation direction [Sheeley and Wang, 2001; Owens et al., 2011a]. We found very good 

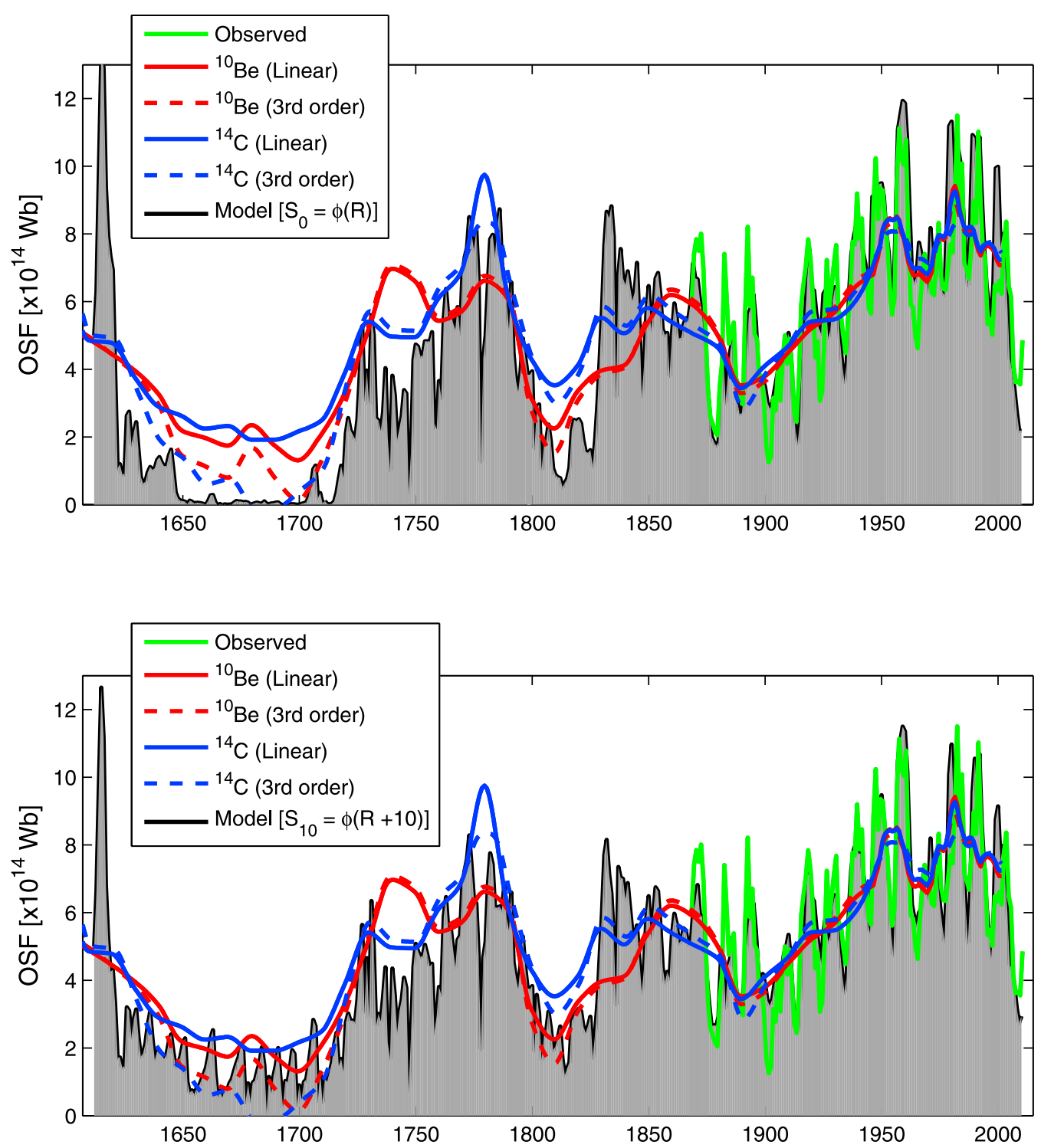

Figure 5. (top) The dark shading shows the annual mean OSF reconstruction using an OSF source term proportional to $R\left(S_{0}=\phi R\right)$ and a cyclic loss term, $\chi_{0}$. Combined spacecraft and geomagnetic annual means of OSF observations are shown in green. Ten year averages of cosmogenic isotope estimates of OSF are shown in blue (from ${ }^{14} \mathrm{C}$ ) and red (from ${ }^{10} \mathrm{Be}$ ), with solid and dashed lines showing linear and third-order fits to OSF, respectively. (bottom) The same OSF reconstruction, but with $S_{10}=\phi(R+10)$ and $\chi_{10}$, to mimic the behavior of CME rates over the last two solar minima.

agreement between $\chi$ and potential field source surface estimates of HCS tilt and local HCS inclination, over the three solar cycles for which data are available. There was also general agreement between $\chi$ and five solar cycles of HCS tilt computed from modeling GCR fluxes [Alanko-Huotari et al., 2007]. Thus we find strong support for OSF loss being related to HCS orientation. By extension, HCS tilt and hence polarity reversal must be unaffected by the large cycle-to-cycle variations in sunspot number, OSF and solar cycle length. It seems that while the magnitude of the solar magnetic field varies considerably over decadal time scales, the spatial distribution of magnetic flux, as manifest by both the HCS orientation [Jiang et al., 2010] and the average latitude of sunspots [Owens et al., 2011b], has a regular cyclic variation.
[26] The next step was to exploit the cyclic behavior of OSF loss, ascribed to HCS inclination, to extend the Owens et al. [2011a] model of OSF further into the past. The PFSSderived OSF loss function was replaced with a general cyclic OSF loss function, but otherwise the model was unchanged. The model OSF obtained in this manner matches the observed OSF back to 1868 extremely well. There is also general agreement with the longer-term variation in OSF inferred from cosmogenic isotope data.

[27] The largest discrepancy between the OSF observations and our model reconstruction is during the most recent solar minimum, where the model with $S_{0}$, a source term proportional to $R$, predicts a larger drop in OSF than observed. This is related to the underestimate in OSF production at times of low sunspot number. Comparing CME rate with $R$, we deduce that 


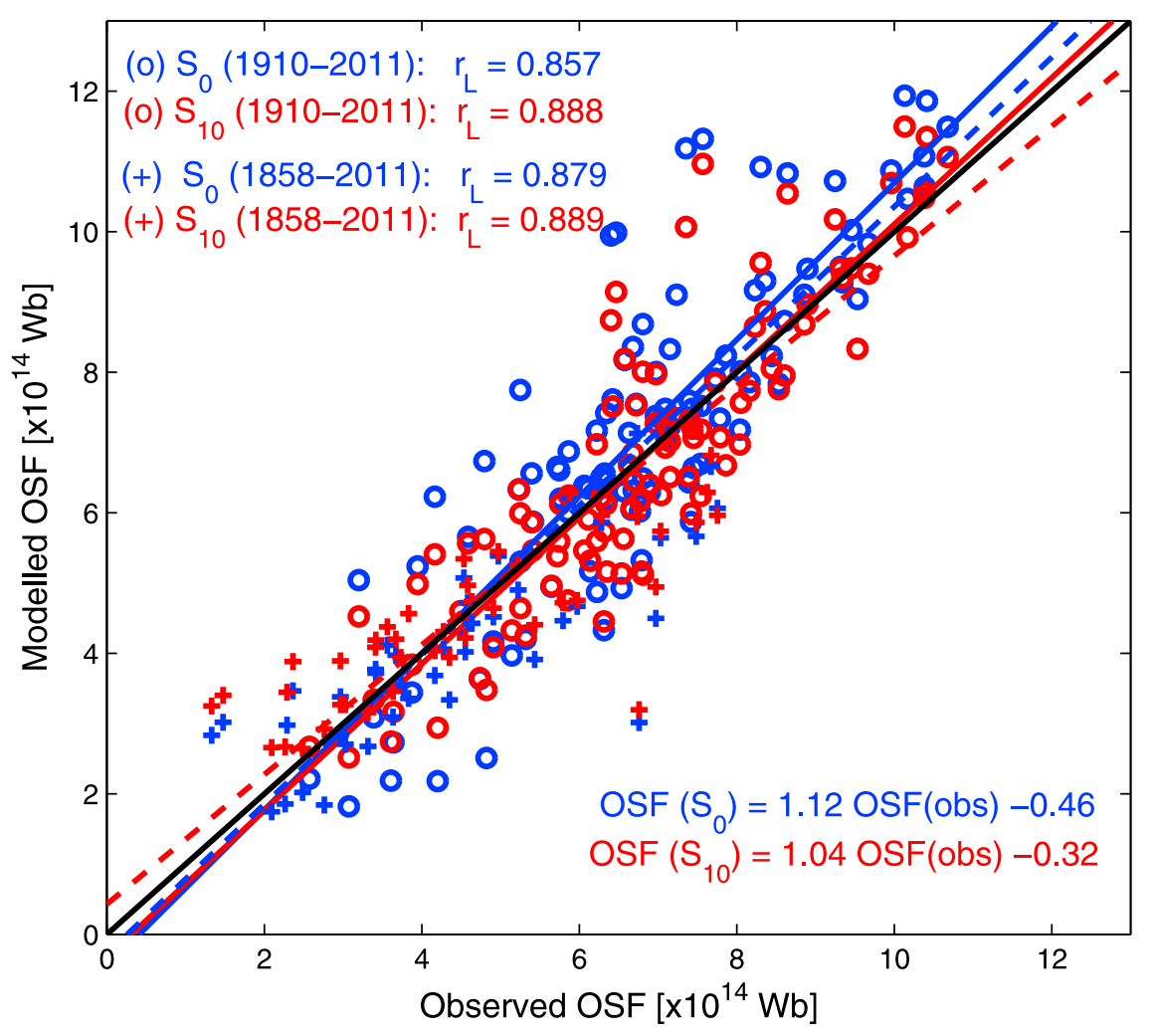

Figure 6. The observed and modeled OSF. Model results using the $S_{0}$ and $S_{10}$ source terms are shown in blue and red, respectively. Crosses (circles) show data over the whole 1858-2011 interval (1910-2011, when the uncertainty in the observed OSF is significantly reduced), with linear best fits shown as dashed (solid) lines.

the OSF production does still occur at $R=0$. Incorporating this new source term, $S_{10}$, into the OSF reconstruction produces very similar values to the original reconstruction, except at a few key times. First, it provides a much better match with the recent solar minimum and Dalton minimum (around 1810). Secondly, there is nonzero OSF during the Maunder Minimum, with a very clear solar cycle variation. There is evidence in GCR records of a solar cycle variation in the heliospheric modulation parameter during this period [Beer et al., 1998], which would require such cycling throughout the Maunder Minimum.

[28] Cosmogenic isotope data suggest the 25 year mean of near-Earth heliospheric magnetic field, $B$, fell to $1.80 \pm 0.59 \mathrm{nT}$ by 1700 , the end of the Maunder M3inimum [Steinhilber et al., 2010]. Lockwood and Owens [2011] used this value with the [Lockwood et al., 2009b] polynomial fit of signed open solar flux, $F_{S}$, against $B$ from geomagnetic and space age interplanetary data (constrained so that $B=0$ if $F_{S}=0$ ) to estimate $F_{S}=(0.48 \pm 0.29) \times 10^{14} \mathrm{~Wb}$ at this time. This corresponds to an unsigned OSF, used in this study, of $2 F_{S}=(0.96 \pm 0.58) \times 10^{14} \mathrm{~Wb}$. From Figure 5, the thirdorder polynomial fits to the cosmogenic isotope data used here give values slightly below this uncertainty range (dashed lines) whereas the linear fits (solid lines) give values slightly above it. The modeled OSF values are within the range for the $S_{10}$ source term but not for $S_{0}$. Thus our modeled OSF for the end of the Maunder Minimum is consistent with the empirical estimate of [Lockwood and Owens, 2011] if the OSF source term $S_{10}$ is used. This gives continued emergence of OSF during the Maunder Minimum which would be necessary to maintain the continued cycling of the HCS tilt in the Maunder Minimum also inferred here. Wang and Sheeley [2003] also suggest that solar magnetic fields were strong enough during the Maunder Minimum to reverse the global polarity of the Sun.

[29] Finally, we note that it is possible that the CME contribution to the total OSF source term at solar minimum has varied over time. However, Figure 3 (top) shows that the CME rate during the recent, lower, solar minimum was about the same as during the prior minimum, despite the lower OSF. This implies that solar minimum $\mathrm{CME}$ rate may be independent of OSF, which in turn suggests that the CME rate observed during recent solar cycle minima is also the $\mathrm{CME}$ rate that existed throughout the Maunder Minimum.

[30] Acknowledgments. We thank T. Hoeksema of Stanford University for WSO magnetograms and HCS tilt data.

[31] Philippa Browning thanks the reviewers for their assistance in evaluating this paper.

\section{References}

Alanko-Huotari, K., I. G. Usoskin, K. Mursula, and G. A. Kovaltsov (2007), Cyclic variations of the heliospheric tilt angle and cosmic ray modulation, Adv. Space Res., 40, 1064-1069, doi:10.1016/j. asr.2007.02.007.

Altschuler, M. A., and G. Newkirk Jr. (1969), Magnetic fields and the structure of the solar corona, Sol. Phys., 9, 131-149.

Barnard, L., M. Lockwood, M. A. Hapgood, M. J. Owens, C. J. Davis, and F. Steinhilber (2011), Predicting space climate change, Geophys. Res. Lett., 38, L16103, doi:10.1029/2011GL048489.

Beer, J., S. Tobias, and N. Weiss (1998), An active Sun throughout the Maunder Minimum, Sol. Phys., 181, 237-249. 
Connick, D. E., C. Smith, and N. A. Schwadron (2011), Interplanetary magnetic flux depletion during protracted solar minimums, Astrophys. J., 727, 8, doi:10.1088/0004-637X/727/1/8.

Crooker, N. U., J. T. Gosling, and S. W. Kahler (2002), Reducing heliospheric flux from coronal mass ejections without disconnection, J. Geophys. Res., 107(A2), 1028, doi:10.1029/2001JA000236.

Ferreira, S. E. S., and M. S. Potgieter (2003), Modulation over a 22-year cosmic ray cycle: On the tilt angles of the heliospheric current sheet Adv. Space Res., 32(4), 657-662, doi:10.1016/S0273-1177(03)00360-0.

Gopalswamy, N., S. Yashiro, G. Michalek, G. Stenborg, A. Vourlidas, S. Freeland, and R. Howard (2008), The SOHO/LASCO CME catalog, Earth Moon Planets, 104, 295-313.

Gosling, J. T., D. N. Baker, S. J. Bame, W. C. Feldman, and R. D. Zwick (1987), Bidirectional solar wind electron heat flux events, J. Geophys. Res., 92, 8519-8535.

Hathaway, D. H., R. M. Wilson, and E. J. Reichmann (2002), Group sunspot numbers: Sunspot cycle characteristics, Sol. Phys., 211, 357-370.

Hoyt, D. V., and K. H. Schatten (1998), Group sunspot numbers: A new solar activity reconstruction, Sol. Phys., 181, 491-512.

Jiang, J., R. Cameron, D. Schmitt, and M. Schüssler (2010), Modeling the Sun's open magnetic flux and the heliospheric current sheet, Astrophys. J., 709, 301-307, doi:10.1088/0004-637X/709/1/301.

Krivova, N. A., L. Balmaceda, and S. K. Solanki (2007), Reconstruction of solar total irradiance since 1700 from the surface magnetic flux, Astron. Astrophys., 467, 335-346, doi:10.1051/0004-6361:20066725.

Lockwood, M. (2006), What do cosmogenic isotopes tell us about past solar forcing of climate?, Space Sci. Rev., 125, 95-109, doi:10.1007/s11214006-9049-2.

Lockwood, M. (2010), Solar change and climate: An update in the light of the current exceptional solar minimum, Proc. R. Soc. A, 466, 303-329, doi:10.1098/rspa.2009.0519.

Lockwood, M., and C. Fröhlich (2008), Recent oppositely directed trends in solar climate forcings and the global mean surface air temperature. II. Different reconstructions of the total solar irradiance variation and dependence on response time scale, Proc. R. Soc. A, 464, 1367-1385, doi:10.1098/rspa.2007.0347.

Lockwood, M., and M. Owens (2009), The accuracy of using the Ulysses result of the spatial invariance of the radial heliospheric field to compute the open solar flux, Astrophys. J., 701, 964-973, doi:10.1088/0004$637 X / 701 / 2 / 964$.

Lockwood, M., and M. J. Owens (2011), Centennial changes in the heliospheric magnetic field and open solar flux: The consensus view from geomagnetic data and cosmogenic isotopes and its implications, J. Geophys. Res., 116, A04109, doi:10.1029/2010JA016220.

Lockwood, M., R. Stamper, and M. N. Wild (1999), A doubling of the Sun's coronal magnetic field during the past 100 years, Nature, 399 437-439, doi:10.1038/20867.

Lockwood, M., M. Owens, and A. P. Rouillard (2009a), Excess open solar magnetic flux from satellite data: 1 . Analysis of the third perihelion Ulysses pass, J. Geophys. Res, 114, A11103, doi:10.1029/2009JA014449.

Lockwood, M., A. P. Rouillard, and I. D. Finch (2009b), The rise and fall of open solar flux during the current grand solar maximum, Astrophys. J., 700, 937-944, doi:10.1088/0004-637X/700/2/937.

Low, B. C. (2001), Coronal mass ejections, magnetic flux ropes, and solar magnetism, J. Geophys. Res., 106, 25,141-25,160.

Macias Fauria, M., A. Grinsted, S. Helama, and J. Holopainen (2012), Persistence matters: Estimation of the statistical significance of paleoclimatic reconstruction statistics from autocorrelated time series, Dendrochronologia, doi:10.1016/j.dendro.2011.08.003, in press.

McCracken, K. G. (2007), Heliomagnetic field near Earth, 1428-2005, J. Geophys. Res., 112, A09106, doi:10.1029/2006JA012119.

McCracken, K. G., and J. Beer (2007), Long-term changes in the cosmic ray intensity at Earth, 1428-2005, J. Geophys. Res., 112, A10101, doi: $10.1029 / 2006 \mathrm{JA} 012117$.

Muscheler, R., F. Joos, J. Beer, S. A. Müller, M. Vonmoos, and I. Snowball (2007), Solar activity during the last $1000 \mathrm{yr}$ inferred from radionuclide records, Quat. Sci. Rev., 26, 82-97, doi:10.1016/j.quascirev.2006.07.012.

Owens, M. J., and N. U. Crooker (2006), Coronal mass ejections and magnetic flux buildup in the heliosphere, J. Geophys. Res., 111, A10104 doi:10.1029/2006JA011641.
Owens, M. J., and N. U. Crooker (2007), Reconciling the electron counterstreaming and dropout occurrence rates with the heliospheric flux budget, J. Geophys. Res., 112, A06106, doi:10.1029/2006JA012159.

Owens, M. J., C. N. Arge, N. U. Crooker, N. A. Schwadron, and T. S. Horbury (2008a), Estimating total heliospheric magnetic flux from single-point in situ measurements, J. Geophys. Res., 113, A12103, doi:10.1029/2008JA013677.

Owens, M. J., N. U. Crooker, N. A. Schwadron, T. S. Horbury, S. Yashiro, H. Xie, O. C. St Cyr, and N. Gopalswamy (2008b), Conservation of open solar magnetic flux and the floor in the heliospheric magnetic field, Geophys. Res. Lett., 35, L20108, doi:10.1029/2008GL035813.

Owens, M. J., N. U. Crooker, and M. Lockwood (2011a), How is open solar magnetic flux lost over the solar cycle?, J. Geophys. Res., 116, A04111, doi:10.1029/2010JA016039.

Owens, M. J., M. Lockwood, C. J. Davis, and L. Barnard (2011b), Solar cycle 24: Implications for energetic particles and long-term space climate change, Geophys. Res. Lett., 38, L19106, doi:10.1029/2011GL049328.

Schatten, K. H., J. M. Wilcox, and N. F. Ness (1969), A model of interplanetary and coronal magnetic fields, Sol. Phys., 9, 442-455.

Sheeley, N. R., Jr., and Y.-M. Wang (2001), Coronal inflows and sector magnetism, Astrophys. J. Lett., 562, L107-L110, doi:10.1086/338104.

Solanki, S. K., M. Schüssler, and M. Fligge (2000), Evolution of the Sun's large-scale magnetic field since the Maunder Minimum, Nature, 408, 445-447, doi:10.1038/35044027.

St. Cyr, O. C., et al. (2000), Properties of coronal mass ejections: SOHO LASCO observations from January 1996 to June 1998, J. Geophys. Res., 105, 18,169-18,185.

Steinhilber, F., J. Beer, and C. Fröhlich (2009), Total solar irradiance during the Holocene, Geophys. Res. Lett., 361, L19704, doi:10.1029/ 2009GL040142.

Steinhilber, F., J. A. Abreu, J. Beer, and K. G. McCracken (2010), Interplanetary magnetic field during the past 9300 years inferred from cosmogenic radionuclides, J. Geophys. Res., 115, A01104, doi:10.1029/ 2009JA014193

Usoskin, I. G. (2008), A history of solar activity over millennia, Living Rev. Sol. Phys., 5, 3.

Usoskin, I. G., S. K. Solanki, M. Schüssler, K. Mursula, and K. Alanko (2003), Millennium-scale sunspot number reconstruction: Evidence for an unusually active Sun since the 1940s, Phys. Rev. Lett., 91(21), 211101, doi:10.1103/PhysRevLett.91.211101.

Usoskin, I. G., K. Alanko-Huotari, G. A. Kovaltsov, and K. Mursula (2005), Heliospheric modulation of cosmic rays: Monthly reconstruction for 1951-2004, J. Geophys. Res., 110, A12108, doi:10.1029/ 2005JA011250.

Vaquero, J. M., M. C. Gallego, I. G. Usoskin, and G. A. Kovaltsov (2011), Revisited sunspot data: A new scenario for the onset of the Maunder Minimum, Astrophys. J. Lett., 731, L24, doi:10.1088/2041-8205/731/2/L24.

Vieira, L. E. A., and S. K. Solanki (2010), Evolution of the solar magnetic flux on time scales of years to millenia, Astron. Astrophys., 509, A100, doi:10.1051/0004-6361/200913276.

Wang, Y.-M., and N. R. Sheeley Jr. (2003), Modeling the Sun's large-scale magnetic field during the Maunder Minimum, Astrophys. J., 591, 1248-1256, doi:10.1086/375449.

Wang, Y.-M., J. L. Lean, and N. R. Sheeley Jr. (2005), Modeling the Sun's magnetic field and irradiance since 1713, Astrophys. J., 625, 522-538, doi:10.1086/429689.

Webb, D. F., and R. A. Howard (1994), The solar cycle variation of coronal mass ejections and the solar wind mass flux, J. Geophys. Res., 99, 4201-4220.

Woods, T. N., W. K. Tobiska, G. J. Rottman, and J. R. Worden (2000), Improved solar Lyman $\alpha$ irradiance modeling from 1947 through 1999 based on UARS observations, J. Geophys. Res., 105, 27,195-27,216, doi:10.1029/2000JA000051.

Yashiro, S., N. Gopalswamy, G. Michalek, O. C. St. Cyr, S. P. Plunkett, N. B. Rich, and R. A. Howard (2004), A catalog of white light coronal mass ejections observed by the SOHO spacecraft, J. Geophys. Res., 109, A07105, doi:10.1029/2003JA010282.

M. Lockwood and M. J. Owens, Space Environment Physics Group, Department of Meteorology, University of Reading, Earley Gate, PO Box 243, Reading RG6 6BB, UK. (m.j.owens@reading.ac.uk) 